\title{
Quantitative method for gait pattern detection based on fiber Bragg grating sensors
}

\author{
Lei Ding \\ Xinglin Tong \\ Lie $\mathrm{Yu}$
}




\title{
Quantitative method for gait pattern detection based on fiber Bragg grating sensors
}

\author{
Lei Ding, ${ }^{a}$ Xinglin Tong, ${ }^{\mathrm{a}, *}$ and Lie $\mathrm{Yu}^{\mathrm{b}}$ \\ ${ }^{a}$ Wuhan University of Technology, National Engineering Laboratory for Fiber Optic Sensing Technology, Hongshan District, Wuhan, China \\ ${ }^{b}$ Wuhan Textile University, School of Electronic and Electrical Engineering, Hongshan District, Wuhan, China
}

\begin{abstract}
This paper presents a method that uses fiber Bragg grating (FBG) sensors to distinguish the temporal gait patterns in gait cycles. Unlike most conventional methods that focus on electronic sensors to collect those physical quantities (i.e., strains, forces, pressure, displacements, velocity, and accelerations), the proposed method utilizes the backreflected peak wavelength from FBG sensors to describe the motion characteristics in human walking. Specifically, the FBG sensors are sensitive to external strain with the result that their backreflected peak wavelength will be shifted according to the extent of the influence of external strain. Therefore, when subjects walk in different gait patterns, the strains on FBG sensors will be different such that the magnitude of the backreflected peak wavelength varies. To test the reliability of the FBG sensor platform for gait pattern detection, the gold standard method using force-sensitive resistors (FSRs) for defining gait patterns is introduced as a reference platform. The reliability of the FBG sensor platform is determined by comparing the detection results between the FBG sensors and FSRs platforms. The experimental results show that the FBG sensor platform is reliable in gait pattern detection and gains high reliability when compared with the reference platform. () 2017 Society of Photo-Optical Instrumentation Engineers (SPIE) [DOI: 10.1117/1.JBO.22.3.037005]
\end{abstract}

Keywords: fiber Bragg grating; fiber optic sensors; gait patterns; external strain; backreflected peak wavelength.

Paper 160872R received Dec. 22, 2016; accepted for publication Mar. 14, 2017; published online Mar. $29,2017$.

\section{Introduction}

Fiber Bragg grating (FBG) sensors have undergone a rapid development in the fields of biomechanics, biomedical, and rehabilitation engineering due to their prominent advantages, such as immunity to electromagnetic interference, remote sensing, ease in handling, low cost, small size, and light weight. ${ }^{1-3}$ These distinguishing features make FBG sensors suitable for human body applications as they can be used for gait analysis in walking cycles. The FBG sensors can be alternatives to standard technologies, including electrical strain gauge, piezoelectric, resistive, and other solid-state sensing, measuring physical parameters, and performing high-sensitivity biochemical analysis. ${ }^{1}$

Generally, the FBG sensors are sensitive to external mechanical perturbations with the result that the backreflected peak wavelength will be shifted according to the extent of the influence of external perturbations, which makes the FBG sensors extremely sensitive to axial strain. Therefore, the FBG sensors can satisfy the requirement in sensing a number of physical parameters such as strain, force, and pressure. ${ }^{1}$ Currently, limited results have been achieved by researchers using FBG as a pressure sensor in the fields of biomechanics, biomedical, and rehabilitation engineering. Behrmann et al. developed a fiber optic sensor that incorporated FBGs and microfabricated stainless steel housings for the measurement of tendon forces. The experiment results demonstrated that the sensor was found to be a sensitive and reliable method for acquiring minimally invasive measurements of soft tissue forces. ${ }^{4}$ Guru Prasad et al. described an $a b$ initio design and development of an FBG sensor-based strain sensing plate for the measurement of plantar strain

*Address all correspondence to: Xinglin Tong, E-mail: tongxinglin@whut.edu .cn distribution in the human foot. ${ }^{5}$ In this study, the traditional accelerometers were used to establish a reference platform to test the performance of the designed strain sensing plate based on FBG sensor. Fresvig et al. utilized the FBG sensors as a measurement tool for bone strain by performing measurements both on an acryl tube and on an extracted sample of human femur diaphysis. In their research, strain gauge was selected as a reference to obtain a comparative result. The experiment result showed that no significant differences were found between strain gauges and optic fibers. ${ }^{6}$

Force induced by the human leg can be used for gait analysis, which is a clinically useful tool for quantifying the states of cyclic walking. Functionally, walking is a basic capability that allows humans to pursue their daily lives and function as productive members of society. ${ }^{7}$ Gait analysis during walking is useful in several applications, such as identifying pathologies, evaluating athletes' performance, and rehabilitation engineering. ${ }^{8}$

FBG sensors can be placed on the ball and heel of a human foot to detect the ground contact force (GCF). As described by Kyoungchul and Masayoshi, ${ }^{7}$ each gait pattern has a unique GCF pattern. To the best of our knowledge, only one article ${ }^{9}$ has partially discussed the possible applications of FBG sensing technology in gait analysis. In this study, four sets of optical fiber pressure sensors are embedded in the insole (at the toe, thenar, antithenar, and heel) with the aim of obtaining the state of weight shift and foot rolling. ${ }^{9}$ However, there is no reference platform utilized to test the reliability of the method, and no algorithm or rule is presented to detect the gait patterns in a quantitative way.

This paper aims to test the reliability of FBG sensors for gait pattern detection. To meet this demand, two sets of force-

$1083-3668 / 2017 / \$ 25.00$ @ 2017 SPIE 
sensitive resistors (FSRs) are used to set up the force platform, which is considered the gold standard method for gait analysis. ${ }^{10}$ In addition, the force platform is introduced as a reference, so the reliability can be determined by comparing the detection results between the FBG sensor platform and the force platform.

\section{Experimental Procedures and Methods}

\subsection{Subjects}

This study included seven males and three females (mean age $25.3 \pm 2.3$ years and mean weight $66.3 \pm 5.7 \mathrm{~kg}$ ) with no history of discernable gait abnormalities. The purpose was explained to each subject before they were asked to give their written informed consent for participation in this study. These subjects were selected from the postgraduate students of the National Engineering Laboratory for Fiber Optic Sensing Technology, Wuhan University of Technology, which approved our research.

\subsection{Measurement Principle of FBG Sensors}

As shown in Fig. 1, two sets of FBG sensors (New port Inc., $400 \mathrm{lp} / \mathrm{mm}, 30 \mathrm{~mm} \times 30 \mathrm{~mm}$ ) are twisted separately around the ball and heel of the human foot. The dispersion parameter of each FBG sensor is $-11 \mathrm{ps} / \mathrm{nm}$, and each FBG sensor possesses a high reflectivity of $97 \%$. In addition, the FBG sensor includes a periodic modulation of the refractive index in the core of a single mode fiber optic cable. If the FBG sensor is illuminated with broadband light that meets the Bragg condition, a narrow spectrum is backreflected centered around the Bragg wavelength $\lambda_{\mathrm{B}}$, which depends on the periodic variation $\Lambda$ of the FBG and the effective index $n_{\text {eff }}$. ${ }^{1}$ This condition can be described as follows:

$\lambda_{\mathrm{B}}=2 n_{\mathrm{eff}} \Lambda$.

Light that does not meet the Bragg condition transmits through the structure. In this paper, the reflected range of $\lambda_{\mathrm{B}}$ is from 1277 to $1292 \mathrm{~nm}, n_{\text {eff }}$ is about 1.45 , and $\Lambda$ is $\sim 443 \mathrm{~nm}$.

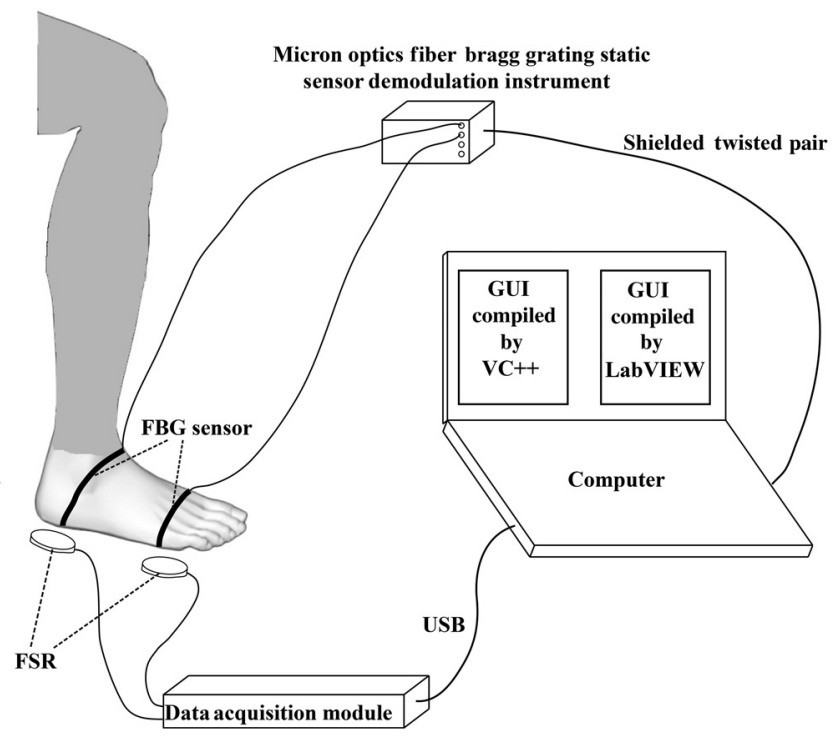

Fig. 1 Schematic diagram of gait pattern detection systems.
The range of index modulations is in the $10^{-5}$ level, while the grating lengths range from 3 to $5 \mathrm{~nm}$.

When the strain in an FBG sensor changes, the backreflected wavelength will shift such that the stain can be measured through the wavelength shift. Moreover, the length of the FBG sensor changes with the axial strain, and the change quantity of the FBG sensor changes with the axial strain is written as $\Delta l$. As a result, this situation produces a change in the grating period (i.e., $\Lambda$ ). Meanwhile, the axial strain induces a change in $n_{\text {eff }}$, the effective index of the grating. Therefore, according to Eq. (1), the change in the center wavelength due to axial strain can be stated as

$\Delta \lambda_{\mathrm{B}}=2\left(\Lambda \frac{\partial n_{\mathrm{eff}}}{\partial l}+n_{\mathrm{eff}} \frac{\partial \Lambda}{\partial l}\right) \Delta l$.

The FBG sensor used in this work is highly sensitive to axial strain, and small variability in the length of the FBG will lead to measurable change in the backreflected light spectrum. In addition, the temperature variability would also cause the length changes of FBG sensor. ${ }^{11}$ Therefore, the work is carried out in a constant temperature laboratory, so the FBG sensors are only subjected to the axial strain. To determine the Bragg wavelength $\lambda_{\mathrm{B}}$, a micron optics (MOI) FBG static sensor demodulation instrument, as seen in Fig. 1, is used to capture the central wavelengths of the backreflected signal.

The central wavelengths are transmitted into a notebook computer through a shielded twisted pair with a Registered Jack 45 (RJ45) connector. One graphical user interface (GUI) compiled by LabVIEW is utilized to store the central wavelengths in synchronization with the computer clock. In addition, the central wavelength data from FBG sensor are filtered by Butterworth low pass filter with a cut-off frequency of $5 \mathrm{~Hz}$.

\subsection{FSR Calibration}

As shown in Fig. 1, two sets of FSR sensors (LSH-10, LOSON Instrumetation, Nankin, China) were placed separately inside the insole of the ball and heel of the human foot. The FSRs were utilized to capture the magnitude variations of GCFs in the plantar area, while the GCF data were collected at a frequency of $1000 \mathrm{~Hz}$ using a data acquisition module (National Instruments) with a universal serial bus (USB). The measurement range of each FSR is from 0 to $200 \mathrm{~kg}$, and each FSR possesses a high accuracy of $\pm 0.5 \%$ full scale. As the respondence of FSR shows the best linearity to the GCF exerted by the human foot in different walking postures, standard load cells $(5,10,20,25,50$, 100 , and $200 \mathrm{~kg}$ ) are used to calibrate the FSR. Because the output signal of each FSR is a week microvoltage, an amplification circuit is integrated to amplify the output signal of each FSR to 0 to $5 \mathrm{~V}$, which correlates with the measured mass of 0 to $200 \mathrm{~kg}$.

The GCF data collected by the data acquisition module are transmitted into the notebook computer through the USB connector. Another GUI compiled by Visual C++ 6.0 is used to store the GCF data in synchronization with the computer clock. Moreover, the GCF data from FSRs are filtered by Butterworth low pass filter with a cut-off frequency of $10 \mathrm{~Hz}$.

\subsection{Description of Walking Experiments}

After sensor calibrations, the experiments are carried out to test the reliability of FBG sensors for gait pattern detection. For 
reference platform, two sets of FSRs are mounted in different foot contact points such as the ball and the heel. For test platform, one FBG sensor is located in a twisted way around the ball of the human foot, while the other is mounted around the heel of the human foot. Then, each subject performed 10 trials including at least 50 gait cycles each time at a time. The first five trials were performed in a straight line, while the other five trials were performed in a curved line.

\subsection{FBG Wavelength Shift During Different Walking Poses}

As reported by Li et al., the FBG sensor is also sensitive to temperature changes, so the entire experiments are conducted in a laboratory with constant temperature under a controlled condition. ${ }^{2}$ To determine the wavelength change of the FBG, the Bragg wavelength shift was recorded using an MOI FBG static sensor demodulation instrument. It is quantitatively illustrated in Table 1 where the original and after-mounted central wavelengths of two FBG sensors are presented. Figure 2 shows the FBG wavelength changes during human motion process. It can be seen that the FBG spectrum would be slightly shifted away from its original central wavelength as the FBG sensor is laid still on the human body. In addition, the FBG spectrum would continue to be shifted when subjects begin to take a step. Figure 3 shows the curve of FBG central wavelength change during gait cycles. The cyclic characteristic of the curve suggests that the central wavelength changes correlate to human walking and exhibit the possibility of application to gait pattern detection.

Table 1 Central wavelength $(\mathrm{nm})$ of the FBG sensor.

\begin{tabular}{lcc} 
No. & Original & $\begin{array}{c}\text { After-mounted on } \\
\text { human body }\end{array}$ \\
\hline 1 & 1284.627 & 1284.629 \\
2 & 1284.812 & 1284.814 \\
\hline
\end{tabular}

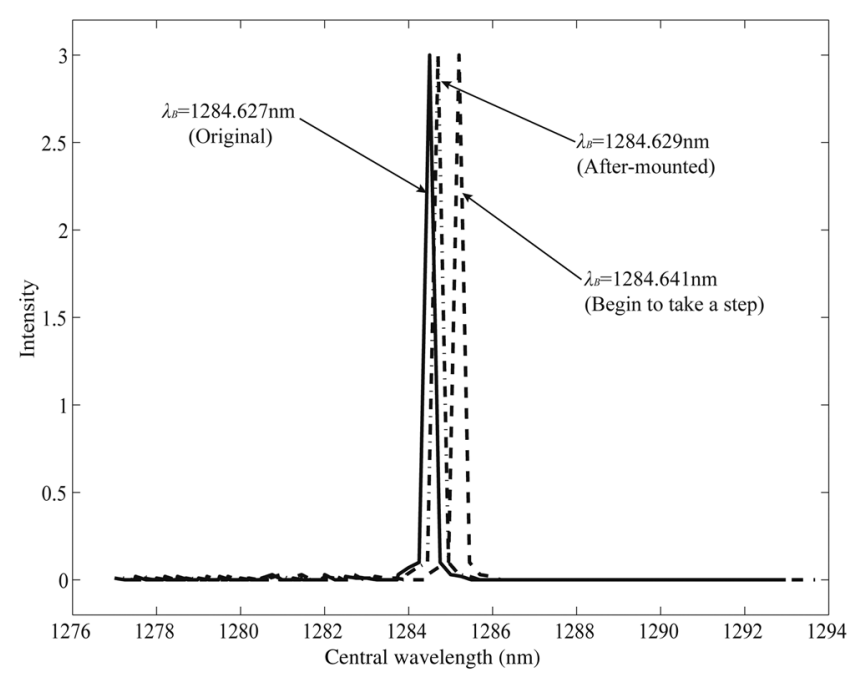

Fig. 2 Central wavelength shift on different motion conditions.

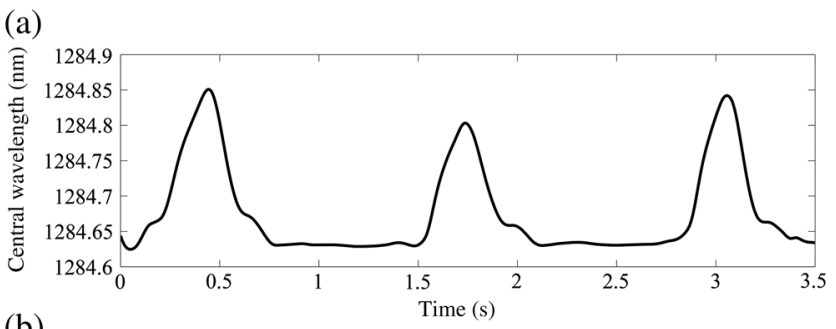

(b)

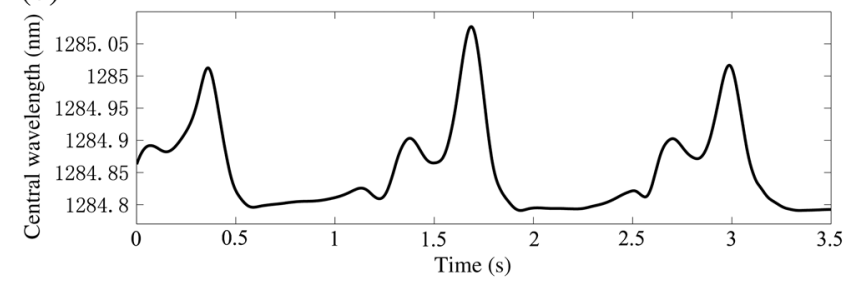

Fig. 3 Curve of central wavelength from FBG sensors: (a) mounted on the ball and (b) mounted on the heel.

\subsection{Gait Patterns Detection Algorithms}

In human walking, a whole gait cycle can be divided into stancephase and swing-phase. The transitions between gait phases are gait events. ${ }^{12}$ Specifically, the transition from swing-phase to stance-phase is the gait event of heel-strike, while the transition from stance-phase to swing-phase is the gait event of heel-off. Stance-phase is defined as when the human foot is in full contact with the ground. Swing-phase is defined as when the human foot totally leaves the ground. Heel-strike is defined as when the heel of the human foot contacts the ground but the ball leaves the ground. Heel-off is defined as when the heel of the human foot rises from the ground but the ball touches the ground. In this paper, two types of sensor platforms are employed to detect these gait patterns, and the detection methods are presented in the following.

\subsubsection{Detection algorithm for force platform}

The FSRs measure the GCFs from the ball and heel in the walking cycle. However, the GCFs cannot be directly transformed into specific gait patterns. Generally, the basic method is to apply a threshold to the vertical component of the GCF such that the GCFs can be divided into on-ground and off-ground statuses. The on-ground status indicates that the human leg touches the ground, while the off-ground status indicates that the human leg leaves the ground. The knowledge of the human leg touching or leaving the ground can lead to the gait pattern detection.

As reported by Smith, ${ }^{13} 80 \%$ of detection errors are due to the failure of the FSRs signal not reaching the threshold value. Threshold setting is the key point for gait pattern detection and attracted many researchers' interest. Different threshold setting methods have been proposed in the literature, such as a fixed threshold of $0,{ }^{14} 2.5 \mathrm{~N},{ }^{15}$ or $10 \mathrm{~N},{ }^{16}$ a proportion of body weight, ${ }^{11}$ or a calculated threshold through the GCF extremes. ${ }^{17,18}$ When the threshold is obtained through one of these methods, the GCFs can be divided into on-ground and off-ground statuses, which is described as

$G= \begin{cases}1, & f \geq T \\ 0, & f<T\end{cases}$

where $f$ is the GCF and $T$ is the threshold. For $G$, " 1 " indicates an on-ground status and "0" indicates an off-ground status. 
Table 2 Rules of gait pattern detection algorithm for force platform.

\begin{tabular}{lccc} 
Heel & Ball & \multicolumn{2}{c}{ Gait patterns } \\
\hline 1 & 1 & Gait phase & Stance-phase \\
0 & 0 & Gait phase & Swing-phase \\
1 & 0 & Gait event & Heel-strike \\
0 & 1 & Gait event & Heel-off \\
\hline
\end{tabular}

Note: "1" for on-ground status and "0" for off-ground status.

Then, based on the detection rules in Table 2, the gait patterns can be analyzed through the divided statuses of GCFs from the heel and ball.

\subsubsection{Detection algorithm for FBG sensor platform}

The detection rules are based on the status division of the sensor signal. Similar to the reference platform, a threshold is set to divide the central wavelength curve into on-ground and offground statuses.

$S= \begin{cases}1, & \lambda_{\mathrm{B}} \geq T_{\mathrm{F}} \\ 0, & \lambda_{\mathrm{B}}<T_{\mathrm{F}}\end{cases}$

where $\lambda_{\mathrm{B}}$ is the central wavelength from the FBG sensors and $T_{\mathrm{F}}$ is the threshold with the same unit of $\lambda_{\mathrm{B}}$. Here, for $S$, " 1 " indicates a on-ground status and " 0 " indicates an off-ground status.

As described by Yu et al., the threshold value can be computed in an equation, given the maximum and minimum values of sensor signals. ${ }^{10}$ Hence, $T_{\mathrm{F}}$ in Eq. (4) can be obtained as follows:

$T_{F}=\lambda_{\mathrm{Bmin}}+\alpha\left(\lambda_{\mathrm{Bmax}}-\lambda_{\mathrm{Bmin}}\right)$

where $\alpha$ is a proportional factor that adjusted the threshold (i.e., $T_{\mathrm{F}}$ ) as a fraction of the difference between the maximum and the minimum of the central wavelengths (i.e., $\lambda_{\mathrm{B}}$ ) for the compensation of interindividual strain variability. $\lambda_{\mathrm{B} \text { max }}$ and $\lambda_{\mathrm{B} \text { min }}$ are the maximum and the minimum of the central wavelengths, respectively. Furthermore, $T_{\mathrm{F}}$ was calculated for each FBG sensor in each experiment. Finally, the gait patterns can be distinguished based on the rules in Table 3 , according to the status division of the central wavelength from two FBG sensors.

Table 3 Rules of gait pattern detection algorithm for FBG sensor platform.

\begin{tabular}{lccc}
\hline Heel & Ball & \multicolumn{2}{c}{ Gait patterns } \\
\hline 1 & 1 & Gait phase & Stance-phase \\
0 & 0 & Gait phase & Swing-phase \\
1 & 0 & Gait event & Heel-strike \\
0 & 1 & Gait event & Heel-off \\
\hline
\end{tabular}

Note: "1" for on-ground status and "0" for off-ground status

\subsection{Evaluation of the Results}

Many studies ${ }^{19-21}$ in the literature compared their research results with the standard force platforms as reference to investigate the accuracy and reliability of their gait pattern detection systems. Hence, the force platform in this work is chosen as the reference system. The reliability of this study can be determined by comparing the detection results between the reference system and the FBG sensor platform.

\section{Results}

\subsection{Selection of Coefficients}

To determine the reliability of the FBG sensor platform, the golden standard force platform was chosen as the reference. Then, the detection results of the FBG sensor platform were compared with the reference platform.

For the force platform, the threshold value for the status division was determined by the method of defining $5 \%$ of subject body weight. ${ }^{8}$ For the FBG sensor platform, $\alpha$ in Eq. (5) was chosen to be 0.1 .

\subsection{Results of Gait Pattern Detection}

The FBG sensor platform was considerably reliable in gait pattern detection when compared with the golden standard force platform (Table 4). The average reliabilities were $83.50 \%$ and $83.28 \%$ in the first five and other five trials.

The GCFs collected by FSRs from the ball and heel were divided into on-ground status and off-ground status by setting thresholds (Fig. 4). Similarly, the central wavelengths collected by FBG sensors from the ball and heel were divided into onground status and off-ground status (Fig. 5). For the force platform, the gait patterns can be detected through the rules in Table 2 based on the status division of GCFs (Fig. 6). For the FBG sensor platform, the rules in Table 3 can distinguish

Table 4 Reliability of the proposed method.

\begin{tabular}{llcc}
\hline & & \multicolumn{2}{c}{ FBG sensor platform } \\
\cline { 3 - 4 } Subjects & Gender & First five trials (\%) & Other five trials (\%) \\
\hline 1 & Male & 83.54 & 82.53 \\
2 & Male & 86.39 & 83.78 \\
3 & Male & 81.35 & 82.26 \\
4 & Male & 83.26 & 84.29 \\
5 & Male & 82.41 & 83.41 \\
6 & Male & 83.67 & 82.93 \\
7 & Male & 82.69 & 84.42 \\
8 & Female & 84.26 & 81.65 \\
9 & Female & 82.23 & 81.84 \\
10 & Female & 85.19 & 85.64 \\
Average & - & 83.50 & 83.28 \\
\hline
\end{tabular}


(a)

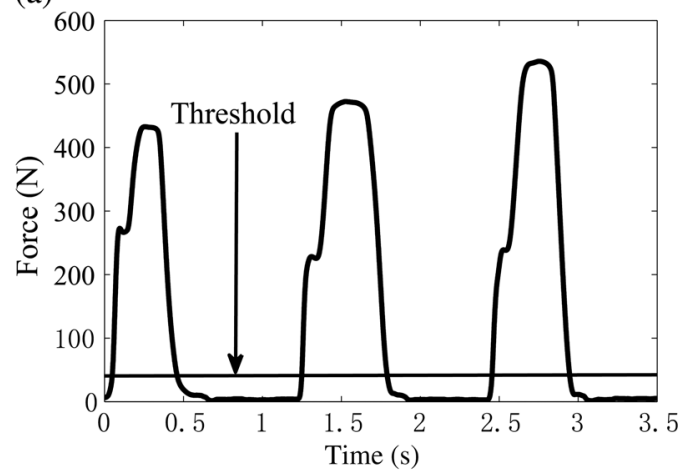

(c)

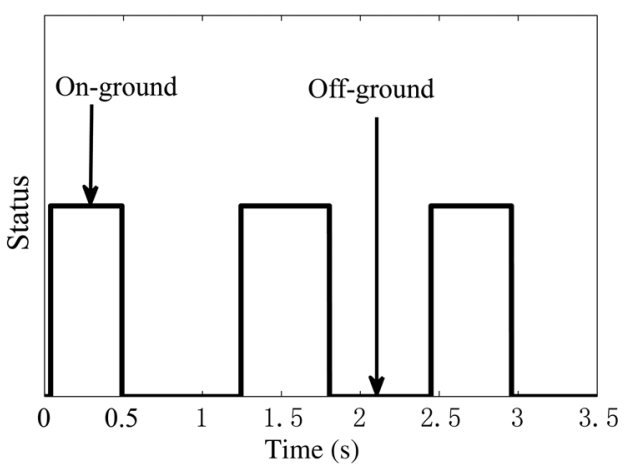

(b)

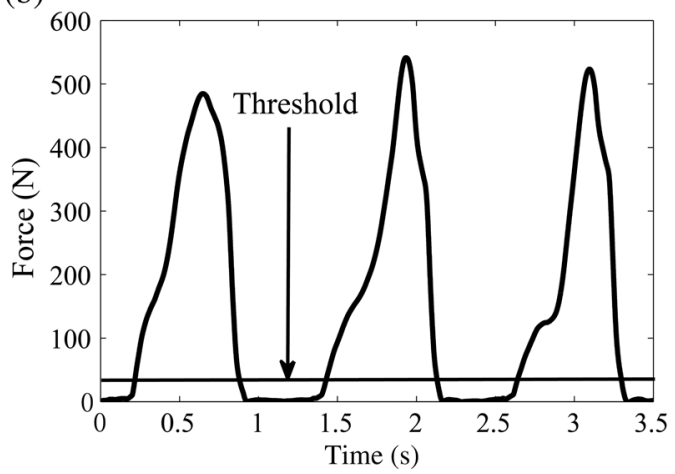

(d)



Fig. 4 A threshold divides the GCF into on-ground and off-ground statuses: (a) GCFs from the ball, (b) GCFs from the heel, (c) status of GCFs from the ball, and (d) status of GCFs from the heel.

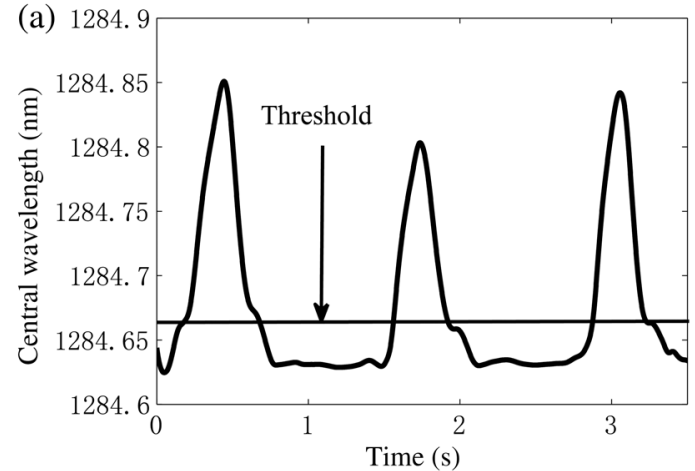

(c)

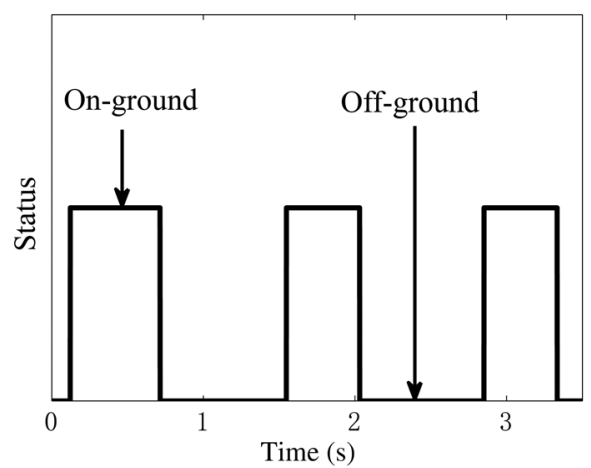

(b)

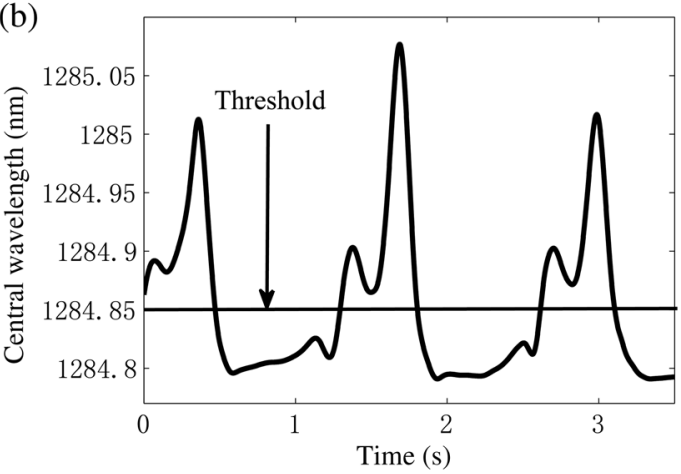

(d)

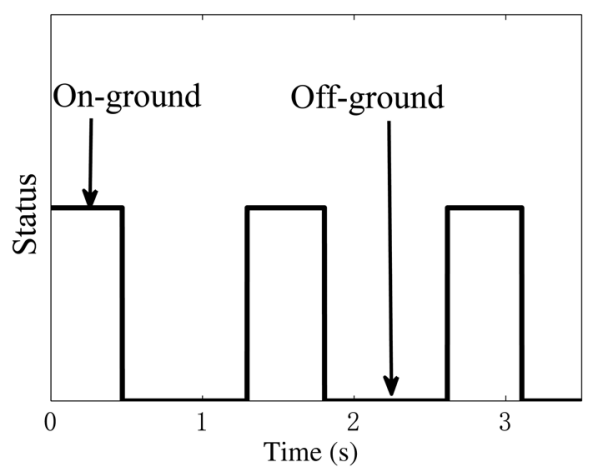

Fig. 5 A threshold divides the central wavelength into tension and no-tension statuses: (a) central wavelengths from the ball, (b) central wavelengths from the heel, (c) status of central wavelengths from the ball, and (d) status of central wavelengths from the heel. 

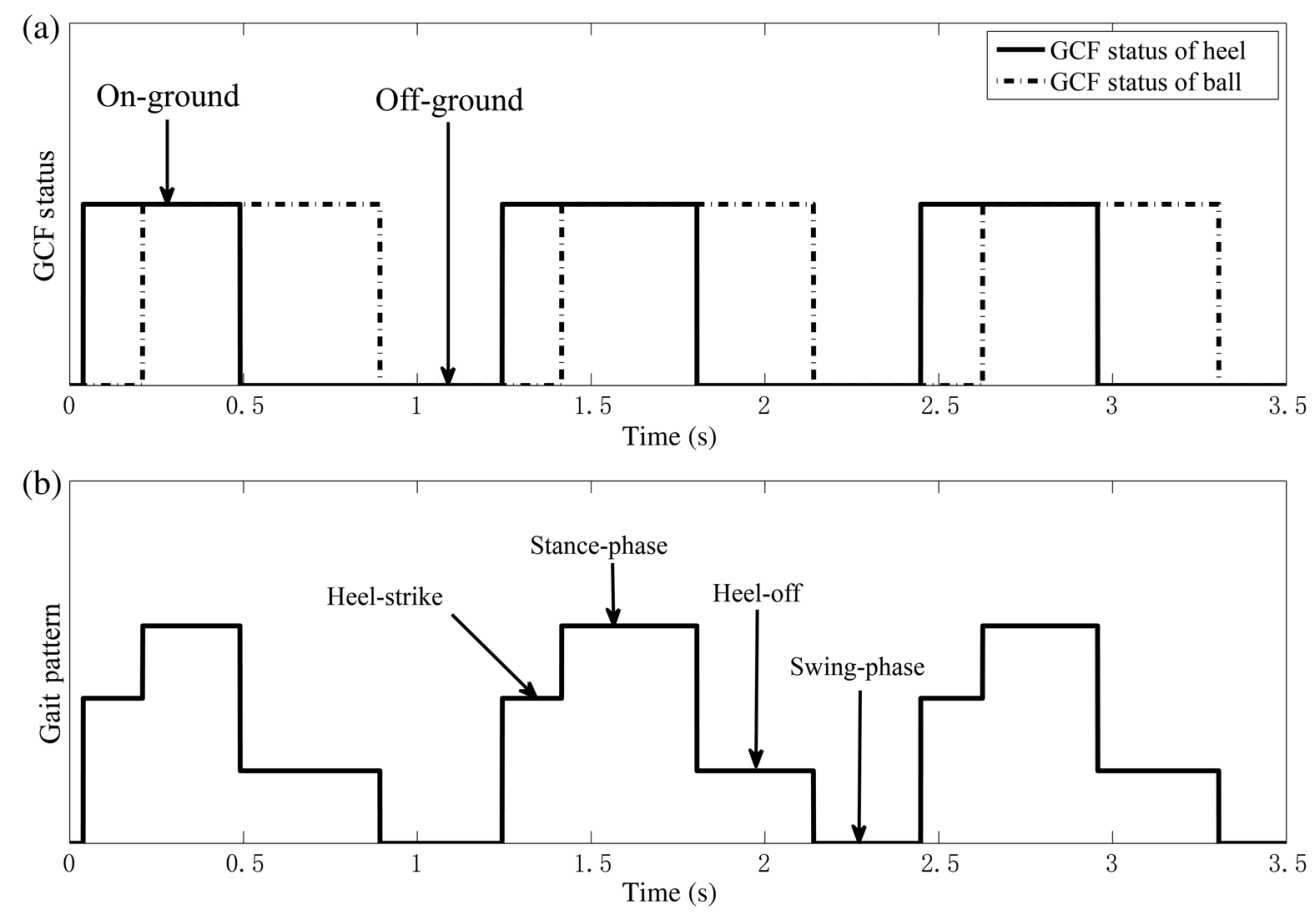

Fig. 6 The gait patterns are distinguished based on the detection rules for force platform.
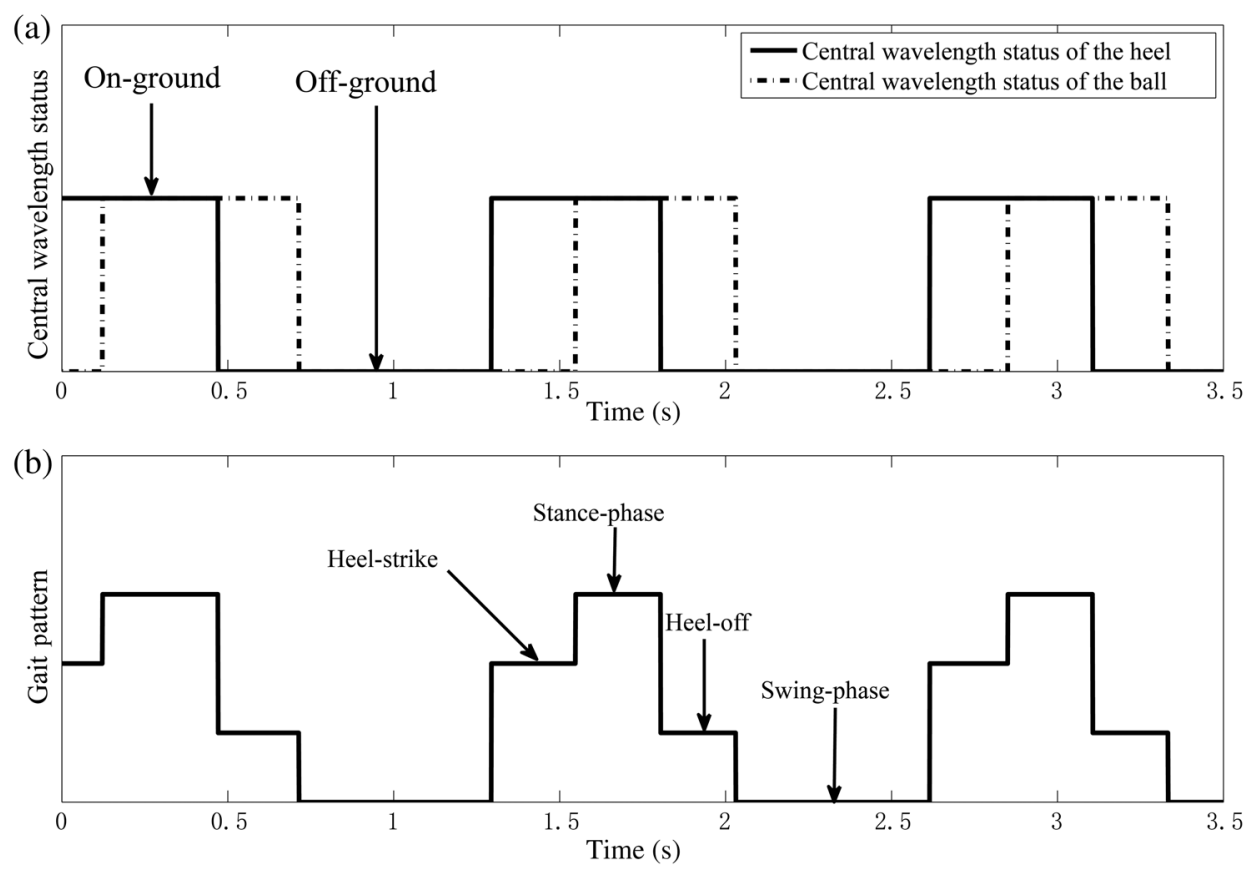

Fig. 7 The gait patterns are distinguished based on the detection rules for FBG sensor platform.

the gait patterns based on the status division of central wavelengths (Fig. 7).

\section{Discussion}

\subsection{Advantages of the Research}

The FBG sensor is easily mounted on human body parts and can measure the tension on the body surface. When a person is in ill health, the tension on the affected part of a patient should be different than that of normal people. Therefore, except for the application to gait pattern detection, the FBG sensor can also be used to monitor people's health.

As applied to gait pattern detection, the FBG sensors need no amplifying circuit, but the FSR does. The central wavelength data can be directly transmitted into a computer using the MOI FBG static sensor demodulation instrument. In some 
experiments, ${ }^{2,3,22}$ the central wavelengths should be calibrated into specific forces or angles. However, the central wavelengths can be directly processed into gait pattern without calibration.

\subsection{Limitations of the Research}

For some body parts, there is little tension in a whole gait cycle such that the central wavelengths of the FBG sensor vary a little. Therefore, the FBG sensor should be located on a human body part where a strong tension occurs.

Sometimes, the subject was sweating in the experiments, so the FBG sensor fell off. As a result, the experiment was stopped until the subject cooled.

\subsection{Future Work}

In fact, the curve of the central wavelengths from the FBG sensor mounted on the shank represents more information, so the subphases of swing can be detected. These subphases include preswing, ${ }^{23}$ toe-off, ${ }^{24}$ and terminal-swing. ${ }^{25}$ Specifically, when the central wavelengths are divided into tension status, the whole leg is swinging in the air and the curve changes back and forth. The curve characteristic correlated with the subphase detection of the swing-phase. ${ }^{26}$ This hypothesis can be affirmed in the future.

\section{Conclusion}

This paper introduces a method that uses a FBG sensor to detect gait patterns. The MOI FBG static sensor demodulation instrument captures the central wavelengths from the FBG sensors. By setting a threshold, the central wavelengths can be divided into tension and no-tension statuses. Based on the detection rules, four types of gait patterns can be distinguished: heelstrike, stance-phase, heel-off, and swing-phase. To test the reliability of the FBG sensor platform, the golden standard force platform is selected as a reference. The reliability of the FBG sensor platform is determined by comparing the detection result between the FBG sensor platform and the reference platform. It is surely concluded that this proposed method is considerably reliable in gait pattern detection.

\section{Disclosures}

The authors have identified the institutional oversight and licensing body that approved the studies. The authors also have confirmed that informed consent was obtained from all subjects. No conflicts of interest, financial or otherwise, are declared by the authors.

\section{Acknowledgments}

This work was supported by the National Nature Science Foundation of China (Grant Nos. 51275373 and 61575148), the National High Technology Research and Development Program of China (863 Project, Grant No. 2015AA043505), and the Key Project of the Nature Science Foundation of Hubei Provincial Government (Grant No. 2014CFA056). The experiment site is provided by China Petrochemical Wuhan Petrochemical Corporation Group, the authors want to thank the workers for giving help.

\section{References}

1. E. Al-Fakih et al., "The use of fiber Bragg grating sensors in biomechanics and rehabilitation applications: the state-of-the-art and ongoing research topics," Sensors 12, 12890-12926 (2012).

2. Y. L. Li et al., "Simultaneous measurement of the temperature and force using a steel cantilever soldered with a partially nickel coated in-fibre Bragg grating," Opt. Commun. 285, 4275-4279 (2012).

3. H. Peng et al., "A novel fiber Bragg grating sensor for weak pressure measurement based on the stokes parameter,' Opt. Fiber Technol. 18, 485-489 (2002).

4. G. P. Behrmann et al., "Fiber optic micro sensor for the measurement of tendon forces," Biomed. Eng. Online 11, 77-93 (2012).

5. A. S. Guru Prasad et al., "Design and development of fiber Bragg grating sensing plate for plantar strain measurement and postural stability analysis," Measurement 47, 789-793 (2014).

6. T. Fresvig et al., "Fibre optic Bragg grating sensors: an alternative method to strain gauges for measuring deformation in bone," Med. Eng. Phys. 30, 104-108 (2008).

7. K. Kyoungchul and T. Masayoshi, "A gait monitoring system based on air pressure sensors embedded in a shoe," IEEE/ASME Trans. Mechatron. 14, 358-370 (2009).

8. C. Senanayake, "Fuzzy logic based implementation of a real-time gait phase detection algorithm using kinematical parameters for walking," in Int. Conf. of Soft Computing and Pattern Recognition, pp. 586-591 (2009).

9. Y. D. Otsuka et al., "Monitoring of plantar pressure in gait based on hetero-core optical fiber sensor," Proc. Eng. 87, 1465-1468 (2014).

10. P. Catalfamo et al., "Detection of gait events using an F-scan in-shoe pressure measurement system," Gait Posture 28, 420-426 (2008).

11. B. Mariani et al., "Quantitative estimation of foot-flat and stance phase of gait using foot-worn inertial sensors," Gait Posture 37, 229-234 (2013).

12. M. M. Skelly and H. J. Chizeck, "Real-time gait event detection for paraplegic FES walking," IEEE Trans. Neural Syst. Rehabil. Eng. 9, 59-68 (2001).

13. B. T. Smith et al., "Evaluation of force-sensing resistors for gait event detection to trigger electrical stimulation to improve walking in the child with cerebral palsy," IEEE Trans. Neural Syst. Rehabil. Eng. 10, 22-29 (2002).

14. A. H. Hansen et al., "A simple method for determination of gait events," J. Biomechan. 35(1), 135-138 (2002).

15. J. C. Wall and J. Crosbie, "Accuracy and reliability of temporal gait measurement," Gait Posture 4, 293-296 (1996).

16. J. Mickelborough et al., "Ennos AR. Validity and reliability of a kinematic protocol for determining foot contact events," Gait Posture 11(1), 32-37 (2000).

17. P. Lopez-Meyer et al., "Automatic detection of temporal gait parameters in poststroke individuals," IEEE Trans. Inf. Technol. Biomed. 15, 594 601 (2011).

18. L. Yu et al., "Adaptive method for real-time gait phase detection based on ground contact forces," Gait Posture 41(1), 269-275 (2015).

19. W. Richard and B. J. Andrews, "Gait event detection for FES using accelerometers and supervised machine learning," IEEE Trans. Rehabil. Eng. 8, 312-319 (2000).

20. M. Ciara et al., "Automatic detection of gait events using kinematic data," Gait Posture 25, 469-474 (2007).

21. K. Aminiana et al., "Spatio-temporal parameters of gait measured by an ambulatory system using miniature gyroscopes," J. Biomechan. 35, 689-699 (2002).

22. N. F. Razali et al., "Fiber Bragg grating for pressure monitoring of full composite lightweight epoxy sleeve strengthening system for submarine pipeline," J. Nat. Gas Sci. Eng. 26, 135-141 (2015).

23. A. Peruzzi et al., "Estimation of stride length in level walking using an inertial measurement unit attached to the foot: a validation of the zero velocity assumption during stance," J. Biomech. 44, 1991-1994 (2011).

24. B. F. Huang et al., "Gait Event detection with intelligent shoes," in Proc. of the 2007 Int. Conf. on Information Acquisition, pp. 579-584 (2007).

25. C. M. Senanayake, "Computational intelligent gait-phase detection system to identify pathological gait," IEEE Trans. Inf. Technol. Biomed. 14, 1173-1179 (2010). 
Ding, Tong, and Yu: Quantitative method for gait pattern detection based on fiber Bragg grating sensors

26. M. Chathuri et al., "Evaluation of gait parameters for gait phase detection during walking," in 2010 IEEE Int. Conf. on Multisensor Fusion and Integration for Intelligent Systems, pp. 127-132 (2010).

Lei Ding is currently a PhD candidate in the Department of Communication Engineering, Wuhan University of Technology, Wuhan, China. He received his BS degree from the Department of Electronic Information Engineering, Naval University of Engineering, Wuhan, China, in 2009, and his MS degree from the Department of Control Engineering, from Wuhan University of Science and Technology, Wuhan, China, in 2011. His current research interests include fiber sensors, biomedical science, and optical coherence tomography.

Xinglin Tong is a professor at the National Engineering Laboratory for Fiber Optic Sensing Technology in Wuhan University of Technol- ogy, Wuhan, China. He received his $\mathrm{PhD}$ degree from the Department of Physics and Electronics, Huazhong University of Science and Technology, Wuhan, China, in 2004. He is the member of the Society of Photo-Optical Instrumentation Engineers. His research interests focus on optical fiber sensing technology and optoelectronics and microelectronic devices and systems.

Lie $\mathrm{Yu}$ is a teacher from Wuhan Textile University in Wuhan city of China. He received his BS degree from Xidian University in 2009, his MS degree from Wuhan University of Science and Technology in 2011, and his PhD from Wuhan University of Science and Technology in 2016. His research interests mainly include the development of biomedical science and rehabilitation robots. 